\title{
Axion-Plasmon Polaritons in Strongly Magnetized Plasmas
}

\author{
H. Terças, ${ }^{*}$ J. D. Rodrigues, and J. T. Mendonça \\ Instituto de Plasmas e Fusão Nuclear, 1049-001 Lisboa, Portugal \\ and Instituto Superior Técnico, 1049-001 Lisboa, Portugal
}

(Received 25 January 2018; revised manuscript received 23 February 2018; published 4 May 2018)

\begin{abstract}
Axions are hypothetical particles related to the violation of the charge-parity symmetry within the strong sector of the standard model, being one of the most prone candidates for dark matter. Multiple attempts to prove their existence are currently performed in different physical systems. Here, we predict that axions may couple to the electrostatic (Langmuir) modes of a strongly magnetized plasma, and show that a new quasiparticle can be defined, the axion-plasmon polariton. The excitation of axions can be inferred from the pronounced modification of the dispersion relation of the Langmuir waves, a feature that we estimate to be accessible in state-of-the-art plasma-based experiments.
\end{abstract}

DOI: 10.1103/PhysRevLett.120.181803

Introduction.-The violation of the charge-parity $(C P)$ symmetry is perhaps one of the most fundamental problems in modern physics [1,2]. Although $C P$-violation is, by construction, inherent to the standard model [3-5], there is no evidence of its manifestation in quantum chromodynamics (QCD). At the origin of the so-called strongCP problem is the anomalous electric dipole moment of the neutron [6], which according to a $C P$-broken QCD calculation would be of the order of $10^{-8}$ e.m., while experiments point towards a $10^{9}$ times larger value. An elegant - and probably the most consensual - way to solve the strong $C P$ problem is the Peccei-Quinn (PQ) mechanism, in which the phase violating the $C P$ symmetry in the QCD Lagrangian is promoted to a complex field $[7,8]$. The corresponding Goldstone pseudoboson is known as the axion, and emerges after breaking the $\mathrm{U}(1)$ symmetry in the gluon coupling term [9].

Axionlike particles (ALPs) are hypothetical particles with an extremely small mass (possibly in the meV range), and they couple very weakly with quarks, leptons, and photons. ALPs have received renewed interest after being indicated as appealing candidates to dark matter in the universe $[10,11]$. Several experiments have been in operation for at least a decade with the goal of observing ALP signatures, using both laboratory and astrophysical observations [12-14]. Unfortunately, most of the observations are too dubious to confirm the existence of ALPs. For example, the PVLAS experiment - originally designed to probe the birefringent properties of the electromagnetic

Published by the American Physical Society under the terms of the Creative Commons Attribution 4.0 International license. Further distribution of this work must maintain attribution to the author(s) and the published article's title, journal citation, and DOI. Funded by SCOAP ${ }^{3}$. vacuum [15] —advanced preliminary results indicating the existence of axions back in 2008, and since then, those findings are the object of controversial debates (see, e.g., $[8,16-18]$ and references therein).

The next generation of experiments based on high-power laser facilities is expected to provide conditions to probe QED physics in parameter regimes that are inaccessible to particle colliders [19]. The ELI experiment will offer the possibility to investigate the effect of the Heiseinberg-Euler vacuum (virtual electron-positon pairs) $[19,20]$ and the quantum recoil due to radiation emission [21]. The wakefield acceleration paradigm has gained much interest as it reveals to be an efficient way to accelerate particles [22-24], and recent studies have exploited such wakefields to produce ALPs in the lab [25-28]. In this Letter, we propose a novel scheme to observe signatures of ALPs in plasma experiments. In the presence of strong magnetic fields, a new quasiparticle is predicted-the axion-plasmon polariton - originating from the hybridization between the axion and the plasma waves. Starting from a PQ-modified electromagnetic theory in the presence of sources, we compute the deformation of the dispersion relation of the electron (Langmuir) waves induced by the axion field. We advance estimates for plasma-based schemes that could demonstrate the existence of axions within the experimentally accessible parameter range.

$P Q$-modified electromagnetism.-A minimal electromagnetic theory can be constructed after integrating out the anomalous axion-gluon triangle, with the effective Lagrangian $[29,30]$

$$
\mathcal{L}=-\frac{1}{4 \mu_{0}} F_{\mu \nu} F^{\mu \nu}-A_{\mu} J_{e}^{\mu}+\mathcal{L}_{\varphi}+\mathcal{L}_{\text {int }},
$$

where $F_{\mu \nu}=\partial_{\mu} A_{\nu}-\partial_{\nu} A_{\mu}$ is the electromagnetic (EM) tensor, $J_{e}^{\mu}$ represents the electron four-current, $\mathcal{L}_{\varphi}=$ $\hbar / c \partial_{\mu} \varphi^{*} \partial^{\mu} \varphi / 2-m_{\varphi}^{2} c /(2 \hbar)|\varphi|^{2}$ is the axion Lagrangian, 
and $\varphi$ is the axion field. The axion mass is given by $m_{\varphi}=\sqrt{z} f_{\pi} m_{\pi} / f_{\varphi}$, where $z=m_{u} / m_{d}$ is the ratio between the up and down quark masses, $m_{\pi}$ is the pion mass, and $f_{\varphi(\pi)}$ is the axion (pion) decay constant [7,9]. The interaction Lagrangian can be constructed as

$$
\mathcal{L}_{\text {int }}=-\frac{g}{4 \mu_{0}} \varphi F_{\mu \nu} \tilde{F}^{\mu \nu}
$$

where $\tilde{F}^{\mu \nu}=\epsilon^{\mu \nu \alpha \beta} F_{\alpha \beta}$ is the dual EM tensor, $\epsilon^{\mu \nu \alpha \beta}$ is the Levi-Civita tensor, and

$$
g=\frac{\alpha_{s}}{2 \pi f_{\varphi}}\left(\frac{E}{N}-\frac{2}{3} \frac{4+z}{1+z}\right)
$$

is the coupling parameter. Here, $\alpha_{s}$ represents the QCD coupling parameter, and $E$ and $N$ represent the EM and the color anomalies of the axion current [31,32]. In Eq. (1), $\varphi=f_{\varphi} \theta$ (with $\theta$ being the $C P$ angle) has dimensions of frequency, while the coupling $g$ has dimensions of time. Here, we have neglected the direct coupling between axions and electrons, a choice that we justify later on. From the Euler-Lagrange equations, and imposing the duality symmetry $\partial_{\mu} \tilde{F}^{\mu \nu}+g \partial_{\mu}\left(\varphi F^{\mu \nu}\right)=\mu_{0} J_{m}^{\nu} / c$, we obtain, in the absence of magnetic currents $\left(J_{m}^{\nu}=0\right)$, the modified Maxwell equations [29]

$$
\begin{aligned}
\boldsymbol{\nabla} \cdot(\mathbf{E}+c g \varphi \mathbf{B}) & =\frac{\rho}{\epsilon_{0}}, \\
\boldsymbol{\nabla} \cdot\left(\mathbf{B}-\frac{g}{c} \varphi \mathbf{E}\right) & =0 \\
\boldsymbol{\nabla} \times(\mathbf{E}+c g \varphi \mathbf{B}) & =-\frac{\partial}{\partial t}\left(\mathbf{B}-\frac{g}{c} \varphi \mathbf{E}\right), \\
\boldsymbol{\nabla} \times\left(\mathbf{B}-\frac{g}{c} \varphi \mathbf{E}\right) & =\frac{1}{c^{2}} \frac{\partial}{\partial t}(\mathbf{E}+c g \varphi \mathbf{B})+\mu_{0} \mathbf{J}_{e},
\end{aligned}
$$

and the Klein-Gordon equation

$$
\left(\square+\frac{m_{\varphi}^{2} c^{2}}{\hbar^{2}}\right) \varphi=-\frac{2 g}{\hbar \mu_{0}} F_{\mu \nu} \tilde{F}^{\mu \nu}=\frac{2 g}{\hbar \mu_{0}} \mathbf{E} \cdot \mathbf{B},
$$

where $\square=\left(1 / c^{2}\right) \partial_{t}^{2}-\nabla^{2}$ is the d'Alembert operator. In a plasma, Eqs. (4), (5) must be closed with the equations for the sources, $\rho=-e\left(n_{e}-n_{i}\right)$ and $\mathbf{u}=-\mathbf{J}_{e} /\left(e n_{e}\right)$, with $n_{e}$ $\left(n_{i}\right)$ denoting the electron (ion) density,

$$
\begin{aligned}
\frac{\partial n_{e}}{\partial t}+\boldsymbol{\nabla} \cdot\left(n_{e} \mathbf{u}\right) & =0, \\
\left(\frac{\partial}{\partial t}+\mathbf{u} \cdot \boldsymbol{\nabla}\right) \mathbf{u} & =-\frac{\boldsymbol{\nabla} P}{m_{e} n_{e}}-\frac{e}{m_{e}}(\mathbf{E}+\mathbf{u} \times \mathbf{B}) .
\end{aligned}
$$

Here, $P=C(T) n_{e}^{\gamma}$ is the electron pressure, $C(T)$ is a temperature-dependent coefficient, and $\gamma$ is the adiabatic exponent [33]. The first row in Eq. (6) can be readily obtained from the first and the fourth lines in (4), while the second one is the momentum equation for a fluid under the Lorentz force. Alternatively, they can be derived by taking the zeroth and the first momenta of the Vlasov equation. We have neglected the ions inertia above, as we are interested in the excitation of axions via electron plasma waves only.

Axion-plasmon polaritons. - Consider a strong, homogeneous magnetic field along the $z$ axis, $\mathbf{B}=B_{0} \mathbf{e}_{z}$. The electrostatic oscillations along the direction of the magnetic field will then provide the EM energy to excite the axion field, as can be seen from Eq. (5). The created axions will then feedback the plasma via the modified Maxwell equations. This effect can be computed by assuming fluctuations around the plasma quasineutrality condition, $n_{e} \sim n_{0}+\tilde{n}$. Taking the decomposition into Fourier modes, $(\tilde{n}, \tilde{\varphi}) \sim e^{i k z-i \omega t}$, and keeping linear terms only, we obtain

$$
\begin{gathered}
\left(\omega^{2}-\omega_{p}^{2}-S_{e}^{2} k^{2}\right) \tilde{n}-i g \frac{e B_{0}}{m_{e}} c k n_{0} \tilde{\varphi}=0, \\
\left(\omega^{2}-\frac{\tilde{m}_{\varphi}^{2} c^{4}}{\hbar^{2}}-c^{2} k^{2}\right) \tilde{\varphi}+2 i g \frac{e c^{4} B_{0}}{\hbar k} \tilde{n}=0,
\end{gathered}
$$

where $S_{e}=\sqrt{\gamma C(T) n_{0}^{\gamma-1} / m_{e}}$ is the electron thermal velocity, $\omega_{p}=\sqrt{e^{2} n_{0} /\left(\epsilon_{0} m_{e}\right)}$ is the plasma frequency, and $\tilde{m}_{\varphi}=\sqrt{m_{\varphi}^{2}+2 \hbar g^{2} B_{0}^{2} /\left(c \mu_{0}\right)}$ is the effective axion mass. Nontrivial solutions to Eq. (7) imply

$$
\left(\omega^{2}-\omega_{\mathrm{pl}}^{2}\right)\left(\omega^{2}-\omega_{\varphi}^{2}\right)-2 g^{2} e n_{0} B_{0} c^{5} \omega_{c} / \hbar=0,
$$

where $\omega_{\mathrm{pl}}^{2}=\omega_{p}^{2}+S_{e}^{2} k^{2}$ and $\omega_{\varphi}^{2}=\tilde{m}_{\varphi}^{2} c^{4} / \hbar^{2}+c^{2} k^{2}$ are the Langmuir and axion bare dispersions, respectively, and $\omega_{c}=e B_{0} / m_{e}$ is the cyclotron frequency. Equation (8) yields the lower $(L)$ and upper $(U)$ polariton modes

$$
\omega_{U, L}^{2}=\frac{1}{2}\left(\omega_{\varphi}^{2}+\omega_{\mathrm{pl}}^{2} \pm \sqrt{\left(\omega_{\mathrm{pl}}^{2}-\omega_{\varphi}^{2}\right)^{2}+4 \Omega^{4}}\right),
$$

where

$$
\Omega=\left(2 g^{2} n_{0} m_{e} c^{5} \omega_{c}^{2} / \hbar\right)^{1 / 4},
$$

represents the Rabi frequency. The dispersion (9) describes the hybridization between the axions and the plasmons. If $\Omega$ is larger than the decay rate $\Gamma$ (to be specified below), a new quasiparticle is formed: the axion-plasmon polariton. As such, if axions are excited by the plasma in the presence of the external magnetic field, then the Langmuir dispersion relation is expected to be strongly modified. In particular, for wave numbers near $\omega_{p} / c$, the Langmuir dispersion abruptly changes from a flat $\left(\sim \omega_{p}\right)$ to a sloped $(\sim c k)$ curve. Physically speaking, it means that the upper polariton mode changes from plasmonlike to axionlike near the crossing 
point $k_{*}=\sqrt{\omega_{p}^{2}-\tilde{m}_{\varphi}^{2} c^{4} / \hbar^{2}} / c$, and conversely for the lower polariton mode. For a typical discharge plasma, $n_{0} \sim 10^{9}-10^{10} \mathrm{~cm}^{-3}$, we estimate the crossing length $\lambda_{*}=2 \pi / k_{*} \sim 1-3 \mathrm{~cm}$. This assures the access to the crossing point with simple experimental techniques, such as Langmuir probes $[34,35]$. The situation may be slightly different for Tokamak $\left(n_{0} \sim 10^{14} \mathrm{~cm}^{-3}\right)$ and solid-state $\left(n_{0} \sim 10^{18} \mathrm{~cm}^{-3}\right)$ plasmas, for which we respectively estimate $\lambda_{*} \sim 0.1 \mathrm{~mm}$ and $\lambda_{*} \sim 1 \mu \mathrm{m}$, implying the use of more sophisticated spectroscopic techniques [36-38]. The features of the axion-polariton modes (9) are summarized in Fig. 1. In order to justify the choice of the minimal model in Eq. (1), we define the effective electron-axion coupling in the plasma $g_{\mathrm{eff}} \sim g_{\varphi e} \hbar^{2} \omega_{p}^{2} /\left(\omega_{c} m_{e}^{2} c^{4}\right) \sim$ $10^{-16}-10^{-11} \mathrm{GeV}^{-1}$, with $g_{\varphi e} \sim 10^{-13}$ denoting the electron-axion Yukawa coupling [39]. Our estimates provide $g_{\text {eff }} / g \sim 10^{-4}-10^{-2}$, which allows us to safely neglect the axion-electron coupling.

Quantization of the theory.-With the aim to unveil the polaritonic character of the avoided crossing in Eq. (9), we proceed to the quantization of the theory. We start by observing that Eq. (7) allows the following decomposition into fast and slow oscillations

$$
\begin{aligned}
& \left(\omega^{2}-\omega_{\mathrm{pl}}^{2}\right) \tilde{n}=\left(\omega-\omega_{\mathrm{pl}}\right)\left(\omega+\omega_{\mathrm{pl}}\right) \tilde{n}, \\
& \left(\omega^{2}-\omega_{\varphi}^{2}\right) \tilde{\varphi}=\left(\omega-\omega_{\varphi}\right)\left(\omega+\omega_{\varphi}\right) \tilde{\varphi} .
\end{aligned}
$$

Since we are interested in the modes that are nearly resonant with the plasma frequency, $\omega \simeq \omega_{p}$, we perform a rotating-wave approximation (RWA) and an inverse Fourier transformation, Eq. (7), to obtain

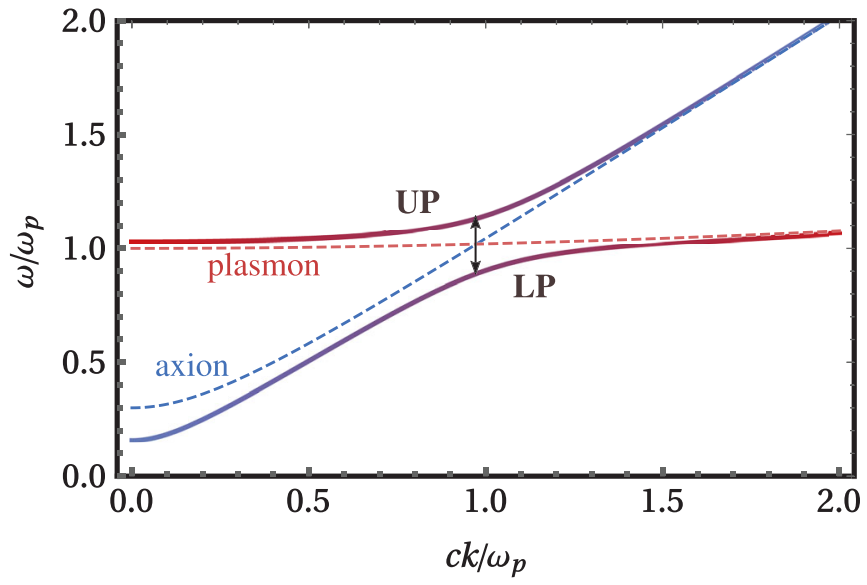

FIG. 1. The dispersion relation of the different modes: axion (blue dashed line), plasmon (red dashed line), lower polariton (LU) and upper polariton (UP). At the crossing point $k_{*} \simeq \omega_{p} / c$, the LP and UP modes are repelled by $2 \Omega$. For illustration, we have set $\Omega=0.5 \omega_{p}$ and $m_{\varphi}=0.3 \hbar \omega_{p} / c^{2}$.

$$
\begin{aligned}
\left(i \frac{\partial}{\partial t}-\omega_{\mathrm{pl}}\right) \tilde{n}-i g \frac{\omega_{c}}{2 \omega_{p}} c k n_{0} \tilde{\varphi} & =0, \\
\left(i \frac{\partial}{\partial t}-\omega_{\varphi}\right) \tilde{\varphi}+i g \frac{e c^{4} B_{0}}{\hbar k \omega_{p}} \tilde{n} & =0,
\end{aligned}
$$

which is valid provided $S_{e} \ll c$, as it is the case of laboratory plasmas. We can represent the plasmon and axion field in terms of bosonic operators $\hat{a}_{k}$ and $\hat{b}_{k}$, obeying the usual commutation relations $\left[\hat{c}_{k}, \hat{c}_{q}^{\dagger}\right]=\delta_{k, q}\left(\hat{c}_{k}=\left\{\hat{a}_{k}, \hat{b}_{k}\right\}\right)$, as

$\tilde{n}(x)=\sum_{k} \mathcal{A}_{k}\left(\hat{a}_{k} e^{i k x}+\hat{a}_{k}^{\dagger} e^{-i k x}\right), \quad \tilde{\varphi}(x)=\sum_{k} \mathcal{B}_{k} e^{i k x} \hat{b}_{k}$,

where $\mathcal{A}_{k}=n_{0} c k / \omega_{p}$ and $\mathcal{B}_{k}=2 m_{e} c^{3} \mathcal{A}_{k} /\left(\hbar k^{2} \omega_{p}\right)$ are some normalization constants. In terms of these operators, Eqs. (12) can be written as Heisenberg equations $i \dot{\hat{c}}_{k}=$ $\left[\hat{c}_{k}, \hat{H}\right]$, associated to the Hamiltonian

$\hat{H}=\sum_{k} \omega_{\mathrm{pl}} \hat{a}_{k}^{\dagger} \hat{a}_{k}+\sum_{k} \omega_{\varphi} \hat{b}_{k}^{\dagger} \hat{b}_{k}+\Omega \sum_{k} \hat{a}_{k}^{\dagger} \hat{b}_{k}+$ H.c.

Full diagonalization can be obtained by introducing the polariton operators $\hat{A}_{k}=u_{k} \hat{a}_{k}-v_{k} \hat{b}_{k}$ and $\hat{B}_{k}=v_{k} \hat{b}_{k}+u_{k} \hat{a}_{k}$, yielding

$$
\hat{H}=\sum_{k} \tilde{\omega}_{L} \hat{A}_{k}^{\dagger} \hat{A}_{k}+\sum_{k} \tilde{\omega}_{U} \hat{B}_{k}^{\dagger} \hat{B}_{k},
$$

with

$$
\tilde{\omega}_{U, L}=\frac{1}{2}\left(\omega_{\varphi}+\omega_{\mathrm{pl}} \pm \sqrt{4 \Omega^{2}+\left(\omega_{\varphi}+\omega_{\mathrm{pl}}\right)^{2}}\right),
$$

being the RWA upper $(U)$ and lower $(L)$ polariton modes. The Hopfield coefficients $u_{k}$ and $v_{k}$ satisfy the normalization condition $\left|u_{k}\right|^{2}+\left|v_{k}\right|^{2}=1$ and read

$$
\begin{aligned}
& u_{k}=\frac{\tilde{\omega}_{U} \omega_{\mathrm{pl}}-\tilde{\omega}_{L} \omega_{\varphi}}{\left(\omega_{\mathrm{pl}}+\omega_{\varphi}\right) \sqrt{\left(\omega_{\mathrm{pl}}-\omega_{\varphi}\right)^{2}+4 \Omega^{2}}}, \\
& v_{k}=\frac{\tilde{\omega}_{U} \omega_{\varphi}-\tilde{\omega}_{L} \omega_{\mathrm{pl}}}{\left(\omega_{\mathrm{pl}}+\omega_{\varphi}\right) \sqrt{\left(\omega_{\mathrm{pl}}-\omega_{\varphi}\right)^{2}+4 \Omega^{2}}} .
\end{aligned}
$$

In Eqs. (14) and (15), we have neglected the counter-rotating terms $\hat{a}_{k}^{\dagger} \hat{b}_{k}^{\dagger}$ and $\hat{a}_{k} \hat{b}_{k}$, a procedure that is well justified provided $\Omega \ll \omega_{\mathrm{pl}}\left(k_{*}\right) \simeq \omega_{p}$. For the conditions we have in mind, we obtain $\Omega / \omega_{p} \sim 10^{-3}-10^{-2}$. However, for sufficiently strong magnetic fields (e.g., at the interior neutron stars or for the plasma of the primordial Universe), the inclusion of the counter-rotating terms may 
be necessary to accurately describe the effect of the plasmonaxion hybridization.

Decay rate.-The second-quantized formalism is particularly helpful to determine the polariton decay rate, a quantity that is crucial to quantify the strength of the plasmon-axion coupling. The mode conversion depicted in Fig. 1 is only observable if the decay rate $\Gamma$ is smaller than the Rabi frequency $\Omega$. This is the so-called strong coupling condition. Two decay mechanisms are considered here. The first one is the radiative decay of the axion into two photons, which occurs at the rate [8]

$$
\Gamma_{\varphi \rightarrow \gamma \gamma}=\frac{g^{2} m_{\varphi}^{3} c^{6}}{64 \pi \hbar^{3}} .
$$

Given the age of the Universe $\left(4.3 \times 10^{17} \mathrm{~s}\right)$, axions with $m_{\varphi} \sim 10 \mathrm{eV}$ should be ruled out [40,41]. Further cosmological implications of axions allow us to limit the scale of the inflation [42], to understand the composition of cold dark matter [43-45]. Moreover, astrophysical considerations exclude axions heavier than $\sim 0.01 \mathrm{eV}[46,47]$. A comprehensive review of the axion cosmology and astrophysics can be found in [48]. The second mechanism is the decay of axions into plasmons, which can be estimated with the help of Fermi's Golden Rule,

$$
\Gamma_{\varphi \rightarrow \mathrm{pl}}=2 \pi \sum_{k, q}\left|\mathcal{M}_{k, q}\right|^{2} \delta\left(\omega_{\varphi}-\omega_{\mathrm{pl}}\right),
$$

where $\mathcal{M}_{k, q}=\Omega \sum_{p}\left\langle k\left|\left(\hat{a}_{p}^{\dagger} \hat{b}_{p}+\hat{a}_{p} \hat{b}_{p}^{\dagger}\right)\right| q\right\rangle$ is the transition amplitude between the states $|k\rangle=\hat{a}_{k}^{\dagger}|0\rangle$ and $|q\rangle=\hat{b}_{q}^{\dagger}|0\rangle$. Considering transitions near the crossing point $\left(\omega_{\varphi} \simeq \omega_{\mathrm{pl}}\right)$, and assuming the axions to be at much lower temperature than the plasmons, we obtain $[49,50]$

$$
\Gamma_{\varphi \rightarrow \mathrm{pl}} \simeq \pi \frac{\Omega^{2}\left[1+n_{a}\left(\omega_{p}\right)\right]}{\sqrt{\omega_{p}^{2}+\tilde{m}_{\varphi}^{2} c^{4} / \hbar^{2}}},
$$

where $n_{a}(x)=\left(\exp \left(x / T_{p}\right)-1\right)^{-1}$ represents the BoseEinstein distribution and $T_{p}$ is the plasmon temperature. From the Mathiessen rule, the total decay rate is $\Gamma=\Gamma_{\varphi \rightarrow \gamma \gamma}+\Gamma_{\varphi \rightarrow \mathrm{pl}} \simeq \Gamma_{\varphi \rightarrow \mathrm{pl}}$, which is valid since we are here discarding higher-order processes such three-wave mixing (nonlinear decay), possibly occurring in extremely dense media or in the presence of shock waves [51]. In a typical discharge plasma, $n_{0} \sim 10^{10} \mathrm{~cm}^{-3}$, with a magnetic field of $B_{0} \sim 1 \mathrm{~T}$, strong coupling can be achieved in a region of parameters that has not yet been excluded by the experiments [52,53]. In Fig. 2, the polariton inclusion or exclusion regions are depicted for different kinds of plasmas. We observe that the most recent data reported by the CAST Collaboration exclude polaritons for couplings $g \gtrsim 10^{-10} \mathrm{GeV}^{-1}$, but they may be produced below this value. The most recent data by the ADMX collaboration

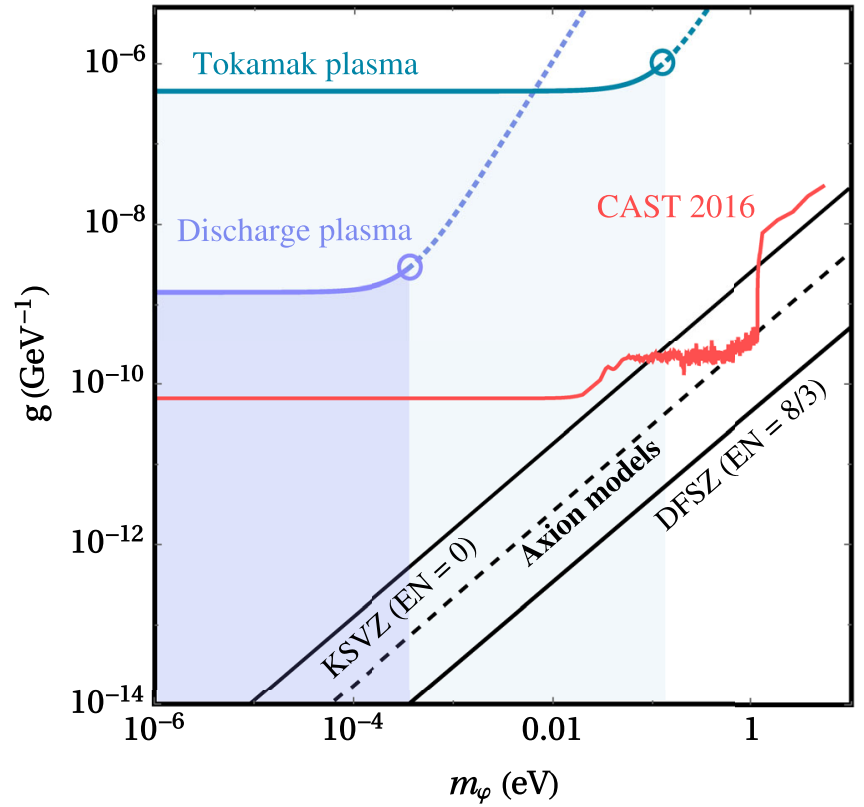

FIG. 2. The axion-plasmon polariton exclusion region. The solid lines delimitate the strong coupling regions (shadowed) for discharge plasmas, $n_{0} \sim 10^{10} \mathrm{~cm}^{-3}$ (light blue line), and Tokamak plasmas, $n_{0} \sim 10^{14} \mathrm{~cm}^{-3}$ (dark blue line). In all cases, we have set $B_{0}=3 \mathrm{~T}$ and neglected the plasmon thermal broadening, $T_{p} \ll \hbar \omega_{p}$. The open circles mark the region $\tilde{m}_{\varphi} \geq \hbar \omega_{p} / c^{2}$, above which the avoided crossing vanishes dynamically. The more recent exclusion region obtained in the CAST experiment [52] (region above the red line) is shown for comparison.

excludes the region $g \gtrsim 10^{-6} m_{\varphi} /(\mathrm{eV})-7.9 \times 10^{-13} \mathrm{GeV}^{-1}$, for $1.97 \leq m_{\varphi} \leq 2.17 \mu \mathrm{eV}$ [54]. Future experiments, such as those projected by the IAXO [55] and MADMAX [56] teams, are designed to probe axions for extremely small masses and axion-photon couplings with unprecedented sensitivity.

Conclusion.-We have shown that the coupling between axions and the plasmons in a strongly magnetized plasma may constitute a mechanism to observe the presence of axions. The most important feature is the avoided crossing between the Langmuir and the axionic Klein-Gordon dispersions near the resonance frequency. The consequence of such a mode repulsion is the changing in character of the Langmuir mode from electrostatic $\left(\omega \sim \omega_{p}\right)$ to electromagnetic $(\omega \sim c k)$. The strong coupling condition has been calculated for different types of plasmas and suggests that laboratory plasmas can be used to probe axions in a region of parameters that is compatible with the state-of-the-art experimental facilities, such as the CAST and ADMX experiments. The present mechanism can be used to investigate a plethora of phenomena involving both cosmological and astrophysical plasmas. The research of axionlike particles in stellar environments has been a subject of intense research, e.g., in understanding their 
impact to the formation of supernovae [57,58], the neutrino signals emitted by supernovae [59], or the enhancing of the spectral hardening of energetic photons [60].

The authors acknowledge FCT-Fundação para a Ciência e Tecnologia (Portugal)—through Grant No. IF/ 00433/2015, and the Doctoral Programme in Physics and Mathematics of Information (DP-PMI) through Grant No. SFRH/BD/52323/2013. The authors also thank Luca Visinelli for enlightening discussions and Igor Irastorza for sharing the most recent data from the CAST Collaboration.

Note added in proof.-We became aware that the most recent data from the ADMX Collaboration, probing with unprecedented sensitivity a previously inaccessible parameter range of the QCD axion, has been meanwhile published in Ref. [61].

*hugo.tercas@tecnico.ulisboa.pt

[1] J. H. Christenson, J. W. Cronin, V. L. Fitch, and R. Turlay, Phys. Rev. Lett. 13, 138 (1964).

[2] A. Alavi-Harati et al. (KTeV Collaboration), Phys. Rev. Lett. 83, 22 (1999).

[3] N. Cabibbo, Phys. Rev. Lett. 10, 531 (1963).

[4] M. Gell-Mann and M. Lévy, Nuovo Cimento 16, 705 (1960).

[5] B. Pontecorvo, Zh. Eksp. Teor. Fiz. 34, 247 (1957) [Sov. Phys. JETP 7, 172 (1958)].

[6] J. M. Pendlebury et al., Phys. Rev. D 92, 092003 (2015).

[7] R. D. Peccei and H. R. Quinn, Phys. Rev. Lett. 38, 1440 (1977).

[8] J. E. Kim and G. Carosi, Rev. Mod. Phys. 82, 557 (2010).

[9] S. Weinberg, Phys. Rev. Lett. 40, 223 (1978).

[10] P. Sikivie, Phys. Rev. Lett. 51, 1415 (1983).

[11] S. J. Asztalos, G. Carosi, C. Hagmann, D. Kinion, K. van Bibber, M. Hotz, L. J. Rosenberg, G. Rybka, J. Hoskins, J. Hwang, P. Sikivie, D. B. Tanner, R. Bradley, and J. Clarke, Phys. Rev. Lett. 104, 041301 (2010).

[12] K. Zioutas et al. (CAST Collaboration), Phys. Rev. Lett. 94, 121301 (2005).

[13] M. Fairbairn, T. Rashba, and S. Troitsky, Phys. Rev. Lett. 98, 201801 (2007).

[14] D. S. Akerib et al. (LUX Collaboration), Phys. Rev. Lett. 118, 261301 (2017).

[15] E. Zavattini, G. Zavattini, G. Ruoso, E. Polacco, E. Milotti, M. Karuza, U. Gastaldi, G. Di Domenico, F. Della Valle, R. Cimino, S. Carusotto, G. Cantatore, and M. Bregant (PVLAS Collaboration), Phys. Rev. Lett. 96, 110406 (2006).

[16] R. N. Mohapatra and S. Nasri, Phys. Rev. Lett. 98, 050402 (2007).

[17] J. Barranco, A. C. Monteverde, and D. Delepine, J. Phys. Conf. Ser. 485, 012035 (2014).

[18] M. Fairbairn, T. Rashba, and S. Troitsky, Phys. Rev. D 84, 125019 (2011).

[19] A. Di Piazza, C. Müller, K. Z. Hatsagortsyan, and C. H. Keitel, Rev. Mod. Phys. 84, 1177 (2012).
[20] M. A. Popovici, I. O. Mitu, G. Cta-Danil, F. Negoi, and C. Ivan, J. Radiol. Prot. 37, 176 (2017).

[21] D. A. Burton and A. Noble, Contemp. Phys. 55, 110 (2014).

[22] S. Mangles, C. Murphy, Z. Najmudin, A. Thomas, J. Collier, A. Dangor, E. Divall, P. Foster, J. Gallacher, C. Hooker, D. Jaroszynski, A. Langley, W. Mori, P. Norreys, F. Tsung, R. Viskup, B. Walton, and K. Krushelnick, Nature (London) 431, 535 (2004).

[23] C. G. R. Geddes, C. Toth, J. van Tilborg, E. Esarey, C. B. Schroeder, D. Bruhwiler, C. Nieter, J. Cary, and W. P. Leemans, Nature (London) 431, 538 (2004).

[24] J. Faure, Y. Glinec, A. Pukhov, S. Kiselev, S. Gordienko, E. Lefebvre, J.-P. Rousseau, F. Burgy, and V. Malka, Nature (London) 431, 541 (2004).

[25] J. T. Mendonça, Europhys. Lett. 79, 21001 (2007).

[26] D. A. Burton and A. Noble, New J. Phys. 20, 033022 (2018).

[27] D. A. Burton and A. Noble, J. Phys. A 43, 075502 (2010).

[28] D. A. Burton, A. Noble, and T. J. Walton, J. Phys. A 49, 385501 (2016).

[29] L. Visinelli, Mod. Phys. Lett. A 28, 1350162 (2013).

[30] F. Wilczek, Phys. Rev. Lett. 58, 1799 (1987).

[31] J. E. Kim, Phys. Rev. Lett. 43, 103 (1979).

[32] M. Dine, W. Fischler, and M. Srednicki, Phys. Lett. 104B, 199 (1981).

[33] F. F. Chen, Introduction to Plasma Physics (Springer, New York, 2012).

[34] R. Grard, A. Pedersen, J.-G. Trotignon, C. Beghin, M. Mogilevsky, Y. Mikhaïlov, O. Molchanov, and V. Formisano, Nature (London) 321, 290 (1986).

[35] E. Chesta, C. M. Lam, N. B. Meezan, D. P. Schmidt, and M. A. Cappelli, IEEE Trans. Plasma Sci. 29, 582 (2001).

[36] M. Burger, D. Pantić, Z. Nikolić, and S. Djeniže, Eur. Phys. J. D 71, 123 (2017).

[37] W.-M. Wang, P. Gibbon, Z.-M. Sheng, and Y.-T. Li, Phys. Rev. Lett. 114, 015001 (2015).

[38] Y. Shi, H. Qin, and N. J. Fisch, arXiv:1712.00517.

[39] E. Armengaud et al., J. Cosmol. Astropart. Phys. 13 (2013) 067.

[40] G. G. Raffelt, J. Phys. A 40, 6607 (2007).

[41] E. Massó and R. Toldrà, Phys. Rev. D 55, 7967 (1997).

[42] M. P. Hertzberg, M. Tegmark, and F. Wilczek, Phys. Rev. D 78, 083507 (2008).

[43] L. Visinelli and P. Gondolo, Phys. Rev. D 80, 035024 (2009).

[44] P. Arias, D. Cadamuro, M. Goodsell, J. Jaeckel, J. Redondo, and A. Ringwald, J. Cosmol. Astropart. Phys. 12 (2012) 013.

[45] P. Gondolo and L. Visinelli, Phys. Rev. Lett. 113, 011802 (2014).

[46] G. G. Raffelt, "Astrophysical axion bounds," in Axions: Theory, Cosmology, and Experimental Searches, edited by M. Kuster, G. Raffelt, and B. Beltrán (Springer-Verlag, Heidelberg, 2008) pp. 51-71.

[47] N. Viaux, M. Catelan, P. B. Stetson, G. G. Raffelt, J. Redondo, A. A. R. Valcarce, and A. Weiss, Phys. Rev. Lett. 111, 231301 (2013)

[48] D. J. Marsh, Phys. Rep. 643, 1 (2016).

[49] H. Terças, S. Ribeiro, M. Pezzutto, and Y. Omar, Phys. Rev. E 95, 022135 (2017). 
[50] H. Terças, S. Ribeiro, and J. T. Mendonça, J. Phys. Condens. Matter 27, 214011 (2015).

[51] H. Bindslev, Plasma Phys. Controlled Fusion 35, 1615 (1993).

[52] V. Anastassopoulos et al. (CAST Collaboration), Nat. Phys. 13, 584 (2017).

[53] J. K. Vogel et al., Phys. Procedia 61, 193 (2015).

[54] L. D. Duffy, P. Sikivie, D. B. Tanner, S. J. Asztalos, C. Hagmann, D. Kinion, L. J. Rosenberg, K. van Bibber, D. B. Yu, and R. F. Bradley, Phys. Rev. D 74, 012006 (2006).

[55] J. K. Vogel et al., Contribution to the 8th Patras Workshop on Axions, WIMPs and WISPs, Chicago, IL, USA, 2012, http://axion-wimp2012.desy.de/e171753/index_eng.html.

[56] A. Caldwell, G. Dvali, B. Majorovits, A. Millar, G. Raffelt, J. Redondo, O. Reimann, F. Simon, and F. Steffen
(MADMAX Working Group), Phys. Rev. Lett. 118, 091801 (2017).

[57] I. Dominguez, M. Giannotti, A. Mirizzi, and O. Straniero, 13th Patras Workshop on Axions, WIMPs and WISPs, Thessaloniki, 2017, http://bib-pubdb1.desy.de/ record/400215.

[58] M. Meyer, M. Giannotti, A. Mirizzi, J. Conrad, and M. A. Sánchez-Conde, Phys. Rev. Lett. 118, 011103 (2017).

[59] T. Fischer, S. Chakraborty, M. Giannotti, A. Mirizzi, A. Payez, and A. Ringwald, Phys. Rev. D 94, 085012 (2016).

[60] D. Montanino, F. Vazza, A. Mirizzi, and M. Viel, Phys. Rev. Lett. 119, 101101 (2017).

[61] N. Du et al. (ADMX Collaboration), Phys. Rev. Lett. 120, 151301 (2018). 